\title{
The importance of recirculation air filtration in containment of SARS-CoV-2 contagion: the role of photocatalytic filters installed in the fan coils
}

\author{
Michele Vio ${ }^{1,{ }^{*}}$, Alessio Gattone ${ }^{2}$ \\ ${ }^{1}$ Consultant - Venezia, Italy \\ ${ }^{2}$ AERMEC - Bevilacqua (VR), Italy
}

\begin{abstract}
In containing the contagion of viral diseases transmitted by air, it is essential to reduce the concentration of elementary dose of airborne nuclei present in inhabited rooms, that is reduced by increasing the renewal outdoor air flow, with the same other factor. The increase in the outdoor air flow, however, finds limits due to energy consumption, the maximum generators power required, the ducts size, which are not always compatible with the spaces available, especially in the case of the renovation of existing buildings. The alternative is the filtration of the recirculated indoor air flow. The paper describes possible strategies. Good filtration can always be possible in all-air systems for cinemas, theatres or supermarkets, or in VAV systems, because the AHUs have dimensions and characteristics such as to allow the housing of any type of filter. VAV systems suffer from the defect of spreading the virus in all rooms, while primary air systems leave it confined only where there is the presence of the infected subject, but filtration is necessary through filters installed directly in the fan coil units. In particular, the paper focuses on the studies carried out in the laboratories of the University of Medicine of Padua for photocatalytic filters and shows the results. The most interesting aspect of this type of filter is that they are effective not only on the finer particles that make up the aerosol, but also on the heavier particles deposited on the surfaces of the room.
\end{abstract}

\section{Introduction}

For airborne diseases, the main infection risk models assume that an infectious person constantly generates a number of infectious quanta over time, with a quantum defined as the dose of airborne droplet nuclei required to cause infection in $63 \%$ of susceptible people [7].

The total number of quanta present in a room at time $t, \mathrm{~N}_{t}$, is obtained from [6]:

$$
N_{t}=\frac{E R_{q} \cdot I}{I V R R}+\left(N_{0}-\frac{E R_{q} \cdot I}{I V R R}\right) \cdot e^{-I V R R \cdot t}
$$

with:

$E R_{\mathrm{q}} \quad$ emission of quanta by the infected subject, $\mathrm{h}^{-1}$;

$I \quad$ number of infected subjects present in the room;

$I V R R$ virus removal rate in the room, $\mathrm{h}^{-1}$;

$N_{0} \quad$ number of quanta present in the room at time $\mathrm{t}=0$.

*Corresponding author: michelevio@studiovio.it; Alessio.Gattone@aermec.com 
$N_{\mathrm{t}}$ does not depend on the room volume, but it strongly depends on the IVRR value. The virus removal rate value is given by three factors [3]:

with:

$$
I V R R=n_{e}+n_{d}+n_{r f}
$$

$n_{\mathrm{e}} \quad$ external fresh air rate through ventilation, $\mathrm{h}^{-1}$;

$n_{\mathrm{d}} \quad$ sum of the the deposition of droplets on the surfaces by sedimentation, $0.24 \mathrm{~h}^{-1}$ [3], and viral inactivation, $0.63 \mathrm{~h}^{-1}[13]$ The total is $0.87 \mathrm{~h}^{-1}$.

$n_{\mathrm{rf}} \quad$ recirculation filtration rate; represents the fictitious increase in the renewal air due to any air filtration through the filters placed on the terminals and / or any other air purifiers, $\mathrm{h}^{-1}$.

The $n_{\mathrm{d}}$ value depends on the disease and is not modifiable. It is necessary to increase the $n_{e}$ and $n_{\mathrm{rf}}$ sum to reduce Nt. The increase in $n_{e}$ generally produces an increase in energy expended for the treatment of the external fresh air and the generators capacity: therefore, $\mathrm{n}_{\mathrm{e}}$ must not exceed the values required for the desired IAQ. It is easier to increase the $n_{\mathrm{rf}}$ value.

The recirculation filtration rate $n_{\mathrm{rf}}$ is given by [3]:

$$
n_{\mathrm{rf}}=\varepsilon_{\mathrm{rf}} \cdot n_{\text {ric }}
$$

with:

$\varepsilon_{\mathrm{rf}} \quad$ filter efficiency on the aerosol particles;

$n_{\text {ric }} \quad$ air recirculation rate inside the room, $\mathrm{h}^{-1}$.

Equation (3) shows that the filter efficiency is not important to minimize IVRR, but the product of the filter efficiency and the recirculation rate. Much better to use a filter with efficiency $=50 \%$ in a terminal capable of moving $n_{\text {ric }}=6 \mathrm{~h}^{-1}$, rather than a $100 \%$ efficiency filter in a $n_{\text {ric }}=1$ h- 1 terminal with: in the first case, the value of $n_{\mathrm{rf}}$ is three times greater, 3 $\mathrm{h}^{-1}$ versus $1 \mathrm{~h}^{-1}$.

\section{Infection risk model}

To estimate airborne transmission risk, the worldwide most used is the model developed by Gammaitoni and Nucci [6]. The G-N model is a variation of the traditional steady-state Wells-Riley model [11]. This model allows to estimate the trend of the individual risk of infection $\mathrm{R}$, as a function of time:

$$
R=1-\exp \left(-I R \int_{T_{p}}^{\left(T_{p}+\tau\right)} \frac{N_{t}}{V} d t\right)
$$

with:

$R \quad$ individual risk of infection for a given $\mathrm{ERq}$;

IR air flow rate of inspired a person, $\mathrm{m}^{3} \cdot \mathrm{h}^{-1}$;

$N_{\mathrm{t}} \quad$ quanta total number in the room at time t, calculated with (1) .;

$V$ room volume of the, $\mathrm{m}^{3}$;

$T_{\mathrm{p}} \quad$ entry time of the exposed person into the room, $\mathrm{h}$;

$\tau \quad$ time spent by the exposed person in the room, $\mathrm{h}$.

$I R$ depends on the level of activity performed. A seated person inhales $0.49 \mathrm{~m}^{3} \cdot \mathrm{h}^{-1}$, $0.54 \mathrm{~m}^{3} \cdot \mathrm{h}^{-1}$ if standing, $1.38 \mathrm{~m}^{3} \cdot \mathrm{h}^{-1}$ in light activity. Knibbs suggests using the medium value equal to $0.6 \mathrm{~m}^{3} \cdot \mathrm{h}^{-1}$ [11]. 
The volume of the room does not affect the number of quanta present in the room, but rather the concentration inhaled by the exposed subjects. With the same $N_{\mathrm{t}}$ value present over time, mainly dependent on IVRR, the infection risk is greater the smaller the volume of the room. This very important aspect was little highlighted in the discussion during the early stages of the pandemic, at least in Italy. For example, supermarkets are safe places thanks to their volume, the quantity of fresh air introduced and the low customer exposure time. In the case of offices, the open spaces are much safer than small rooms, at least from the point of view of the individual risk of infection.

Figure 1 shows, for example, the trend as a function of time of the quanta concentration (left) and the individual infection risk for two different room volume values. It is clear that, for the same number of quanta, the concentration changes and that the individual infection risk decreases almost proportionally to the volume, which is confirmed to be a fundamental element.
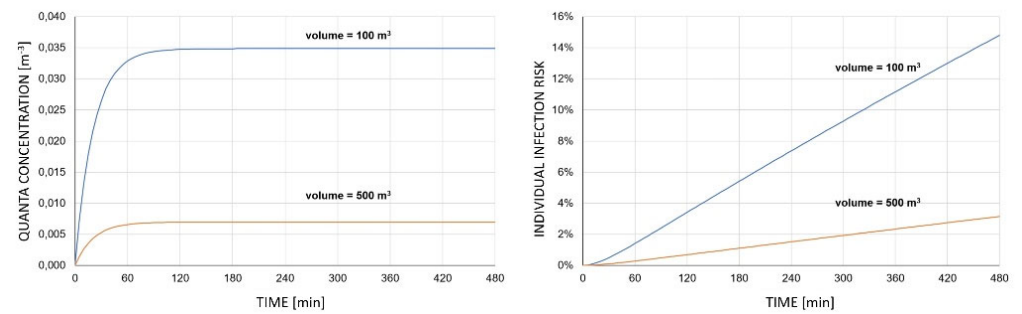

Fig. 1 - trend of the quanta concentration (left) and the individual infection risk (right) for two different room volume values. $I=1, E R \mathrm{q}=10 \mathrm{~h}^{-1},\left(n_{\mathrm{e}}+n_{\mathrm{rf}}\right)=2 \mathrm{vol} \cdot \mathrm{h}^{-1}, I R=0,6 \mathrm{~m}^{3} \cdot \mathrm{h}^{-1}$

\subsection{Collective infection risk}

The collective infection risk of, PIP (Potentially Infected People), expresses the number of exposed people that an infected subject is likely to infect. A collective infection risk greater than 1 implies that the infected person definitely infects at least one exposed person. Collective risk is given by [3]:

$$
P I P=\sum_{1}^{S} R \cdot E
$$

with:

$R \quad$ individual infection risk, calculated with (4);

$E \quad$ number of exposed people;

$S$ number of room or sector of room;

During the first two phases of the SARS-CoV-2 pandemic, in Italy there was great confusion between individual and collective risk. As can be clearly seen from reading the previous paragraphs:

- the individual infection risk, $\mathrm{R}$, depends on the air conditioning system and is totally independent of the number of people occupying the room;

- $\quad$ the collective infection risk, PIP, depends only on the number of people present in the room, or in one of its sectors, each of which is associated with its own individual risk.

It is clear that the collective risk alone is not very significant. For example, it is possible to have a collective risk PIP $=0.5$ both with a single exposed person who has a $50 \%$ probability of being infected, and with 100 exposed persons who have a $0.5 \%$ probability of 
being infected: the collective risk is the same, but for those who have to live or work inside the room in the first case the individual risk is unsustainable while in the second there is a safety situation.

The air conditioning system can only act on the individual infection risk: the building manager can act on the collective risk, allowing more or fewer people to enter it.

\subsection{Limits of infection risk models}

Well - Riley's was a steady-state model: the variation of Gammaitoni - Nucci allows to analyze in detail the trend as a function of time of the phenomena that influence the risk of infection. Both are based on a perfect mixing of the air inside the room, a condition that is almost true only:

- $\quad$ if the infected person is constantly moving around the entire room (for example for a customer in a supermarket);

- $\quad$ in small rooms, where the movements of people and person-to-person respiratory transmission route can be very relevant [1];

- $\quad$ always in the presence of very high air recirculation, which can only be reached in HVAC systems for some special applications.

These limits in the infection risk models do not allow to carefully evaluate the spread of quanta in the space of the room. The results obtained are only an indicative average value, rarely real.

Numerical modelling with CFD can be a powerful alternative as it can avoid these limitations, although care is required for quality assurance [11]. However, an important matter of concern for CFD simulations is the predicted accuracy, which is influenced by geometrical oversimplification of the physical models, grid generation, specification of boundary conditions, and selection of methods of solution. Numerical and modelling errors can occur throughout these processes.

In particular, numerical modelling with CFD only makes sense when all the surrounding conditions are known, first of all the position of the infected person. This is true only in hospital rooms: in investigations for all other cases the position of the infected, asymptomatic person is unknown. Therefore, numerical modelling cannot be applied successfully.

Buonanno and other [3] have proposed a simplified modelling, very useful at an engineering level to understand how the virus moves in space and to better calculate both the individual and collective infection risk. The room is divided into 9 sectors, in each of which a perfect mixing is assumed and the Gammaitoni Nucci model is applied. The virus moves in function of the time on the air flows generated by the position of the diffusers and terminals. Modelling becomes more precise the greater the number of sectors, but it has been seen that 9 sectors are more than enough to help the HVAC system designer make his or her choices.

\section{Virus movement in the room}

The movement of the virus in a room changes depending on whether:

- $\quad$ the virus moves only within a single room: in this case there are differences depending on the type of system, primary air systems or total air systems;

- $\quad$ the virus moves between different rooms: this is the case of VAV systems. 


\subsection{Virus movement in a single room: primary air systems}

In primary air systems, the virus moves inside a room according to the position of the infected person, the speed and direction of the primary air flows and the air exiting the terminals. In particular the type of terminals is important [3]: the cassettes and chilled beams compartmentalize the air flow in a given area of influence, while the fan-coils have a more orientable flow direction. Radiant systems do not compartmentalize the direction of the air at all.

For example, take an open space $20 \mathrm{~m}$ long, $10 \mathrm{~m}$ wide and $2,7 \mathrm{~m}$ high. It accommodates 18 people ( 2 per sector). Primary air distribution occurs along the short side from left to right, with three diffusers and three return air intakes equally spaced. The terminals are three, installed along the upper long side.

Figure 2 shows, using 9-sector modelling, what happens after 8 hours in the hypothesis of the infected person presence, in sector 1, the one at the top left, in the hypothesis that it emits 10 as much $\mathrm{h}^{-1}$ : the figures above show the case of $n_{\text {ric }}=0 \mathrm{~h}^{-1}$, while the ones below show the case of $\mathrm{n}_{\text {ric }}=6 \mathrm{~h}^{-1}$. The external fresh air ratio is always $\mathrm{n}_{\mathrm{e}}=1.7 \mathrm{~h}^{-1}$. The Gammaitoni - Nucci model gives a final quanta number of 3.9, a quanta concentration of $0.007 \mathrm{~m}^{-3}$, an infection individual risk $R=3.2 \%$ and a collective infection risk $P I P=0,55$.

Air flows create a very different reality though the total quanta number is the same. Without air recirculation, the virus moves horizontally very slowly. There are three diffusers and return air intakes, one for each line of sectors. Thus, the virus occupies only a third of the room's volume. The individual risk is much more for the subject who shares sector 1 with the infected person, it goes down but it is still high for the other four people who occupy the two sectors immediately to the right of that of the infected. With recirculation running, the virus spreads throughout the entire volume of the room more quickly. The maximum individual risk, for the person who shares sector 1 with the infected, is lower, while the collective risk increases, because now 17 people are involved and no longer 5 , as in the case of switched off terminals. The situation changes if the infected person is in a different sector.

Quanta concentration per $\mathrm{m}^{3}$

\begin{tabular}{|l|l|l|}
\hline 0,041 & 0,016 & 0,008 \\
\hline & & \\
\hline & & \\
\hline
\end{tabular}

INDIVIDUAL INFECTION RISCK

\begin{tabular}{|c|c|c|}
\hline 0,017 & 0,006 & 0,003 \\
\hline 0,014 & 0,005 & 0,002 \\
\hline 0,011 & 0,004 & 0,002 \\
\hline
\end{tabular}

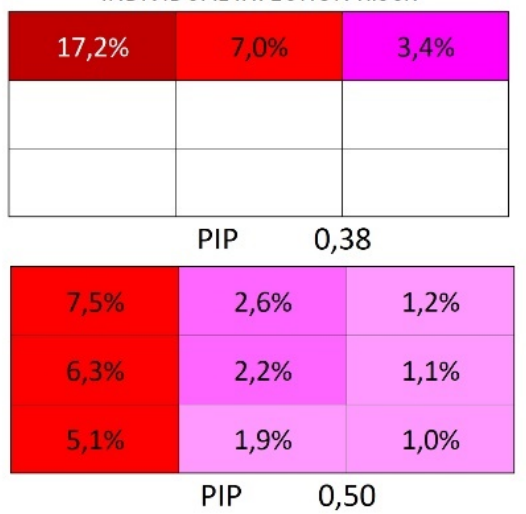

Fig. 2 - Primary air systems: quanta concentration, individual and collective infection risk with infected person in sector 1 , the one at the top left. Above $n_{\text {ric }}=0 \mathrm{~h}^{-1}$, below $n_{\text {ric }}=6 \mathrm{~h}^{-1}$

In any case, a perfect air mixing modelling greatly underestimates the maximum individual risk and overestimates the collective risk, because the quanta concentration is distributed more evenly as the recirculation rate increases. The Gammaitoni - Nucci basic hypothesis of perfect air mixing can only be achieved with an infinite recirculation rate. The 
basic hypothesis of perfect air mixing can only be achieved with an infinite recirculation rate. Consequently, in real cases, as the recirculation rate increases, the maximum individual risk always decreases and the collective risk always increases.

\subsection{Virus movement in a single room: total air systems}

Even with total air systems, the distribution of the virus in the room is more uniform the more the recirculation rate, $\mathrm{n}_{\text {ric }}$, increases. With the same $\mathrm{n}_{\text {ric }}$, the distribution depends on the type of air diffusion (mixing or displacement) and the position of the infected person with respect to the return air intake (Buonanno and others 2021). Figure 3 shows what happens in the same room used in the previous example, with the infected person always positioned in sector 1 , the one at the top left. The figures show the quanta contraction, the individual infection risk and the collective infection risk after 8 hours in the case of ceiling diffusers, one for each sector, and only one return air intake, in the infected sector (above), in that opposite, the one at bottom right (below). The fresh air rate is $n_{e}=1.7 \mathrm{~h}^{-1}$ and the recirculation rate is $n_{\text {ric }}=6 \mathrm{~h}^{-1}$. If the recirculated air intake is in the sector of the infected person, in all the other sectors the distribution is uniform because the mixing between fresh air and recirculated air takes place in the AHU, upstream of the diffusers of each sector.

Due to their characteristics, total air systems distribute the air more evenly and therefore there are fewer differences compared to the perfect air mixture model. However, in the infected sector the individual risk of infection is almost double that predicted by the Gammaitoni-Nucci model. The differences increase if the recirculation rate is reduced: with $n_{\text {ric }}=2 \mathrm{~h}^{-1}$, the maximum risk of individual infection rises from $5.4 \%$ to $8.2 \%$, in the case of return air intake in the sector of the infected person, from $5.1 \%$ to $7.0 \%$, in the case of return air intake in the opposite sector. The collective infection risk drops to $P I P=0.5$ and $P I P=$ 0.51 , respectively.
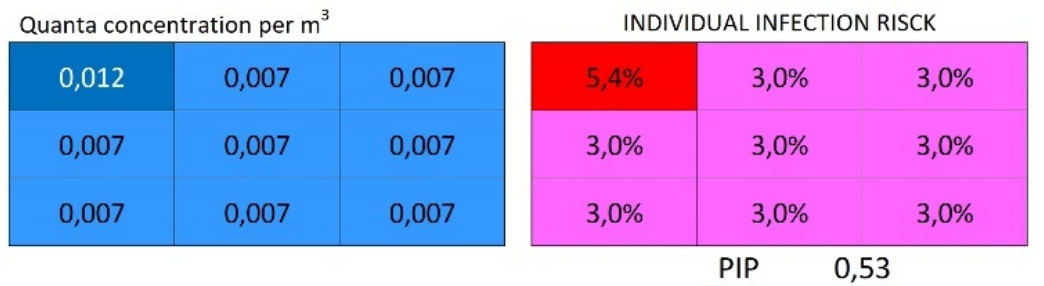

\begin{tabular}{|l|l|l|}
\hline 0,011 & 0,007 & 0,006 \\
\hline 0,008 & 0,007 & 0,006 \\
\hline 0,007 & 0,006 & 0,006 \\
\hline
\end{tabular}

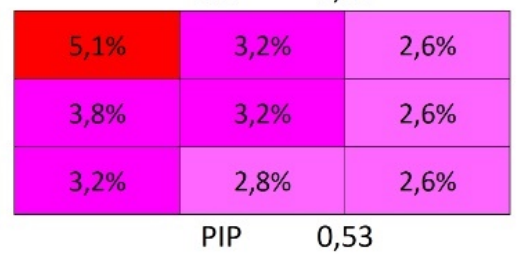

Fig. 3 - Total air system quanta concentration, individual and collective infection risk with infected person in sector 1, the one at the top left. Return air intake in top left sector above and in bottom right sector bellow

\subsection{Virus movement between different rooms: VAV systems}

With VAV systems the quanta concentration distribution is:

- $\quad$ analogous to distribution with total air systems, in the infected person's room 
- completely uniform in the other rooms, because the mixing between fresh air and recirculated air takes place in the AHU, upstream of the diffusers of each room.

The percentage of quanta migrated, from the room where the infected person is present to the other rooms served by the VAV system, depends on the following parameters[3]:

- the ratio between the volume of the room where the infected person is present and the sum of the volumes of all the other rooms, $V_{\mathrm{IPR}} / \Sigma V_{\mathrm{OR}}$;

- $\quad$ the ratio $n_{\mathrm{e}} /\left(n_{\mathrm{e}}+n_{\text {ric }}\right)$ whose value is at most 1 , when the system works with fresh air only. In a VAV system, the total airflow is minimum for most of the year and maximum for only a few hours during the summer. The $\mathrm{n}_{\mathrm{e}}$ value remains constant, while $n_{\text {ric }}$ varies. Consequently, the $n_{\mathrm{e}} /\left(n_{\mathrm{e}}+n_{\text {ric }}\right)$ ratio is maximum for the minimum total airflow and minimum for the maximum total airflow.

All other conditions being equal, the percentage of migrated quanta decreases as both the ratio $V_{\mathrm{IPR}} / \Sigma V_{\mathrm{OR}}$ and the ratio $n_{\mathrm{e}} /\left(n_{\mathrm{e}}+n_{\text {ric }}\right)$ increase. For this reason, a VAV system is more dangerous if it is applied to many small-volume rooms, rather than to a few larger-volume rooms, with the same overall volume served by the system and the number of people present. In addition, a VAV system is more dangerous when it works at maximum total airflow, compared to minimum. The greatest danger is limited to a few hours per year. When it works in free-cooling, it only introduces only external fresh air so the danger is very low.

As an example, let's take 5 rooms with the same dimensions as the one shown in figures 2 and 3 , all connected a VAV system. Then compare the results in the case of 30 rooms with a volume equal to $90 \mathrm{~m}^{3}, 6$ times smaller, each able to accommodate 3 people. In both cases it is assumed that the infected person is positioned near the return air intake.

Table 1 shows the subjects exposed in the case of total presence of people and a reduced presence of $33 \%$ during the pandemic. Table 2 shows the individual risk $\mathrm{R}$ and the collective risk PPC, after 8 hours with the conditions: $E R=10 \mathrm{~h}^{-1}, \mathrm{n}_{\mathrm{e}}=1,7 \mathrm{~h}^{-1}, \mathrm{n}_{\text {ric } \min }=3 \mathrm{~h}^{-1}, \mathrm{n}_{\text {ric } \max }=$ $6 \mathrm{~h}^{-1}$.

The table confirms that the collective infection risk is easily controlled by reducing the number of people present inside the rooms, in the case of a few large rooms, while it is much less so in the case of many small rooms. This reiterates what was said in paragraph 2: the volume of the room is a determining parameter for controlling the spread of the infection.

Slightly different results occur for different positions of the infected person with respect to the return air intake, because the individual risk $R$ in his room changes. The collective infection risk is generally overestimated if the calculation is made considering a perfect air mixing in the infected person room.

Table 1 - Exposed subjects inside the rooms.

\begin{tabular}{|c|c|c|c|c|}
\hline & \multirow{2}{*}{\multicolumn{2}{|c|}{ Infected Person room }} & \multirow{3}{*}{ Other Room } \\
\hline & & & & \\
\hline & & IP sector & Other sectors & \\
\hline \multirow{2}{*}{5 rooms } & $\begin{array}{l}18 \text { persons per } \\
\text { room }\end{array}$ & 1 & 17 & 72 \\
\hline & $\begin{array}{c}12 \text { persons per } \\
\text { room }\end{array}$ & - & 12 & 48 \\
\hline \multirow{2}{*}{30 rooms } & $\begin{array}{c}3 \text { persons per } \\
\text { room }\end{array}$ & - & 2 & 87 \\
\hline & $\begin{array}{l}12 \text { persons per } \\
\text { room }\end{array}$ & - & 1 & 58 \\
\hline
\end{tabular}


Table 2 - Individual Risk $R$ and Collective Risk PIP, after 8 hours. $E R=10 \mathrm{~h}^{-1}, n_{\mathrm{e}}=1,7 \mathrm{~h}^{-1}$,

\begin{tabular}{|c|c|c|c|c|c|c|c|c|}
\hline \multicolumn{9}{|c|}{$n_{\text {ric } \min }=3 \mathrm{~h}^{-1}}$. \\
\hline & & & \multicolumn{2}{|c|}{$\begin{array}{l}\mathrm{R} \text { in Infected } \\
\text { Person room }\end{array}$} & $\mathrm{R}$ & \multicolumn{3}{|c|}{ Collective Risk PIP } \\
\hline & $\mathrm{PpR}$ & Air Flow & $\begin{array}{c}\text { IP } \\
\text { sector }\end{array}$ & $\begin{array}{c}\text { Other } \\
\text { sectors }\end{array}$ & $\begin{array}{l}\text { Other } \\
\text { rooms }\end{array}$ & $\begin{array}{c}\text { IP } \\
\text { rooms }\end{array}$ & $\begin{array}{l}\text { Other } \\
\text { rooms }\end{array}$ & Total \\
\hline \multirow{4}{*}{$\begin{array}{l}5 \\
\text { rooms }\end{array}$} & \multirow{2}{*}{18} & Min & $5,9 \%$ & $2,1 \%$ & $0,68 \%$ & 0,40 & 0,48 & 0,88 \\
\hline & & Max & $5,4 \%$ & $2,0 \%$ & $0,96 \%$ & 0,39 & 0,69 & 1,08 \\
\hline & \multirow{2}{*}{12} & Min & $5,9 \%$ & $2,1 \%$ & $0,68 \%$ & 0,29 & 0,33 & 0,62 \\
\hline & & Max & $5,4 \%$ & $2,0 \%$ & $0,96 \%$ & 0,25 & 0,46 & 0,71 \\
\hline \multirow{4}{*}{$\begin{array}{c}30 \\
\text { rooms }\end{array}$} & \multirow{2}{*}{3} & Min & $35,1 \%$ & $14,5 \%$ & $1,03 \%$ & 0,36 & 0,90 & 1,26 \\
\hline & & Max & $25,8 \%$ & $15,0 \%$ & $1,9 \%$ & 0,33 & 1,62 & 1,95 \\
\hline & \multirow{2}{*}{2} & Min & $35,1 \%$ & $14,5 \%$ & $1,03 \%$ & 0,18 & 0,60 & 0,78 \\
\hline & & Max & $25,8 \%$ & $15,0 \%$ & $1,9 \%$ & 0,16 & 1,08 & 1,24 \\
\hline
\end{tabular}

\section{The effects of recirculated air filtrations}

The contribution of recirculated air must be considered, in an individual and collective infection risk evaluation. According to (2) and (4), it is necessary to increase the $n_{e}$ and $n_{r f}$ sum to reduce the infection risk. The increase in $n_{\mathrm{e}}$ generally produces an increase in energy expended for the treatment of the externa fresh air and in the generators capacity: therefore, $\mathrm{n}_{\mathrm{e}}$ must not exceed the values required for the desired IAQ. It is easier to increase the $n_{\mathrm{rf}}$ value.

According to (3), $\mathrm{n}_{\mathrm{rf}}$ can be increased either by increasing the filter efficiency or by increasing $\mathrm{n}_{\text {ric }}$. It must be remembered that filters generally have a variable efficiency according to the air flow that passes through them: this function has a maximum (Buonanno and others 2021), so $\mathrm{n}_{\mathrm{rf}}$ also has a maximum, for each type of filter. In addition, $\mathrm{n}_{\text {ric }}$ often depends on the capacity that the HVAC system must provide.

\subsection{Total air system in a single room}

The AHUs of the total air systems have sufficient space to house any type of filter and fans with the necessary head for any pressure drop. However, the increased efficiency of the filter leads to more frequent maintenance and higher pressure drops, therefore a higher energy consumption. The use of HEPA filters is not absolutely necessary in civil plants, even during the pandemic phase.

Figure 4 shows the trend of the collective infection risk PIP (left) and its decrease percentage (right) as a filter efficiency function, for the example of figure 3 , in the case of return air intake in the infected person sector.

It is evident how the percentage decrease of the PIP is reduced with the increase of the filter efficiency, the more the lower is $n_{\text {ric }}$. For this reason, it is useless to use HEPA filters: a good level of efficiency between $75 \%$ and $85 \%$ is more than enough.

Rather, it may be worthwhile to design variable air flow systems to work at a certain fixed air flow rate in the event of a pandemic emergency, so as to minimize the risk of infection. 

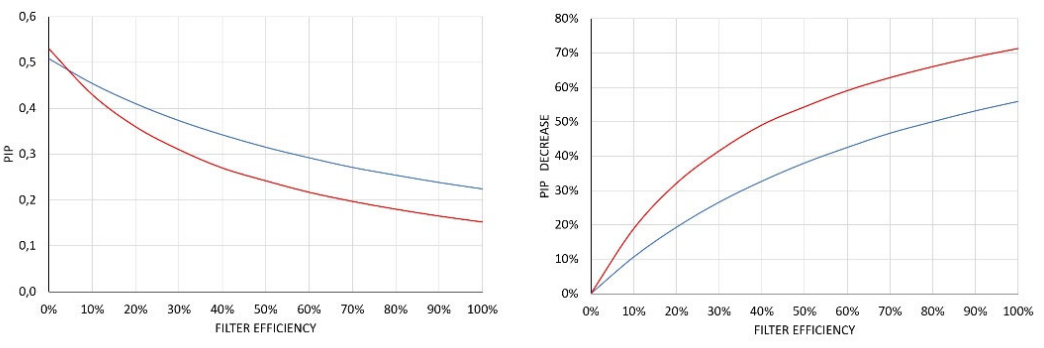

$-n_{\text {ric }}=3 h^{-1} \quad-n_{\text {ric }}=6 h^{-1}$

Fig. 4 - Trend of the collective infection risk PIP (left) and its decrease percentage (right) as a filter efficiency function, for the example of figure 3 , in the case of return air intake in the infected person sector.

\subsection{VAV systems}

The phenomenon, described in the previous paragraph, is even more evident in VAV systems, because two opposite effects come into conflict:

- the increase of $n_{\text {ric }}$ increases the migration of quanta from the room of the infected person to the others;

- $\quad$ the increase of $n_{\text {ric }}$ improves the recirculation filtration rate, reducing the infection risk.

Therefore, the effect of the filter efficiency is a bit lower than that of a Total Air system working on a single room. Figure 5 shows the trend of the collective infection risk PIP (left) and its decrease percentage (right) as a filter efficiency function, for the example of table 5, in the case of 5 rooms and 18 persons per room. The reduction percentage is also very similar for the case with 30 rooms.

The figure confirms that it is not necessary to install HEPA filters: a filtration efficiency between $75 \%$ and $85 \%$ is enough.
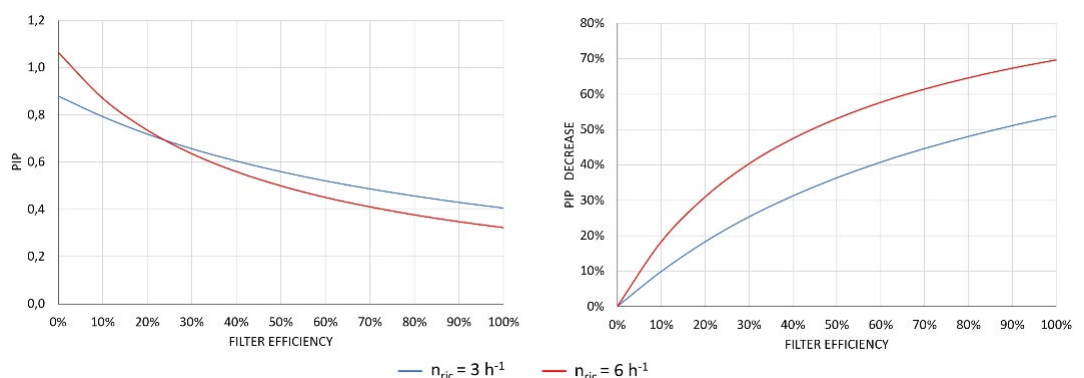

Fig. 5 - Trend of the collective infection risk PIP (left) and its decrease percentage (right) as a filter efficiency function, for the example of Table II, in the case 5 rooms and 18 persons per room.

\subsection{Primary air systems}

From the point of view of air filtration to combat airborne diseases, primary air systems behave differently because:

- $\quad$ it is not possible to use the filters installed in the AHU: the externa fresh air has a quanta concentration equal to 0 ; 
- $\quad$ the filters must be installed directly in the terminals, whenever possible: radiant systems cannot filter the air in any way;

- mechanical filters with a sufficiently high efficiency take up space and produce pressure drops incompatible with the terminal fans.

For these reasons it is necessary to use different filters, such as electrostatic and photocatalytic filters. Figure 6 shows how the individual infection risk, in the sector of the infected person, and the collective infection risk varies, according to the efficiency of the filter installed on the terminals, applied to the example of figure 2 . With a zero-recirculation rate, the risk obviously remains unchanged, because the terminals are off switched and the filters are not crossed by air.

The reduction of individual and collective risks increases as the recirculation rate increases and the efficiency of the filter increases, as is obvious looking at equations (2) and (3). From the point of view of the percentage, the reduction is smaller on the individual risk in the infected person sector compared to which on the collective risk: in the infected person sector the quanta concentration already decreases only as the recirculation rate increases, so the effect of the filter is less.
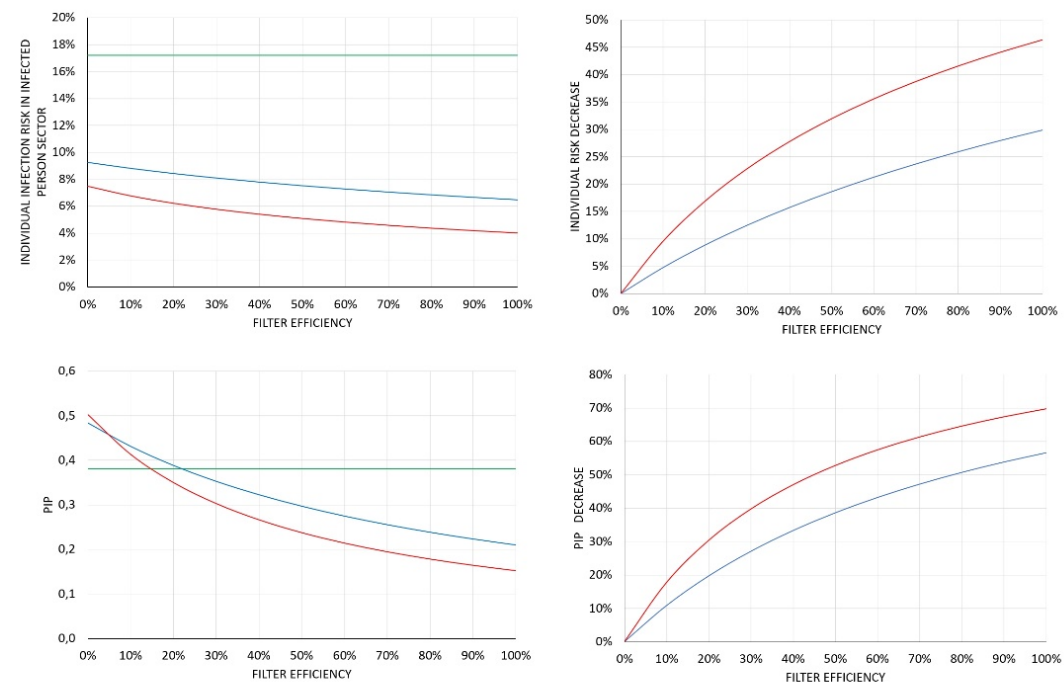

Fig. 6 - Trend of the individual infection risk (above) and collective infection risk PIP (bellow) and its decrease percentage (right) as a filter efficiency function, for the example of figure 2 .

In the pandemic event, it is advisable to maximize the recirculation air flow moved by the terminals as much as possible, in order to reduce the quanta concentration and the risk of infection everywhere. The figure shows how good results are already achieved with filtration efficiency values higher than $60 \%$.

\section{Photocatalysis air cleaning filters}

There is a big difference between air cleaning and air filtration: the air cleaning eliminates viruses, germs and bacteria by killing them, while air filtration temporarily traps them in the 
filters, but does not eliminate their potential danger. Viruses last for a few tens of hours if trapped on surfaces, but bacteria can also reproduce, creating dangerous colonies.

\subsection{Limits of mechanical and electrostatic filters}

Both mechanical filters and electrostatic filters do not clean the air, but retain viruses, germs and bacteria on their surfaces.

Mechanical filters have dimensions and pressure drops that are greater the greater their efficiency. It is easy to install them in AHUs, but much more difficult to use them in terminals such as fan-coils, without affecting performance and dimensions. The efficiency of the mechanical filters increases as their fouling increases, but also the pressure drop increases and therefore the air flow through them decreases. For this reason, they must be replaced with greater frequency, the greater their efficiency. In the case of fan-coils, replacing the filters can represent a danger due to the presence of viruses and bacteria, which can be released back into the room.

Electrostatic filters have much smaller dimensions and pressure drops, so they are suitable for being installed on fan-coils, but their efficiency decreases as their fouling increases. In addition, viruses and bacteria trapped on their surfaces can be released into the room in the event of a electric current failure.

\subsection{The photocatalytic process}

Ultraviolet (UV) irradiation is a widely used method for controlling bioaerosols in indoor environments [8]. The purine and pyrimidine bases of DNA strongly absorb UV irradiation that is between 220 and $300 \mathrm{~nm}$ in wavelength, resulting in modifications or breaks. This sometimes leads to disruption of the genetic function and the death of the exposed organisms. UV irradiation was previously found to have a germicidal and bactericidal effect, which has led many researchers to examine the effects of UV irradiation on the viability of bioaerosols via control of the irradiation dose, the air movement patterns, room configurations, and ambient moisture condition

In a study simulating sunlight on influenza virus aerosols, virus half-life was significantly reduced from $31.6 \mathrm{~min}$ in the dark control group to approximately $2.4 \mathrm{~min}$ in simulated sunlight [12]. While daylight's effect on indoor viruses and SARS-CoV-2 is still unexplored, spectrally tuned electric lighting is already implemented as engineering controls for disinfection indoors. UV light in the region of shorter wavelengths (254-nm UV C) is particularly germicidal, and fixtures tuned to this part of the light spectrum are effectively employed in clinical settings to inactivate infectious aerosols and can reduce the ability of some viruses to survive [5]. Airborne viruses that contain single-stranded RNA (ssRNA) are reduced by $90 \%$ with a low dose of UV light, and the UV dose requirement increases for ssRNA viruses found on surfaces. A previous study demonstrated that $10 \mathrm{~min}$ of UVC light inactivated $9.999 \%$ of CoVs tested, SARS-CoV, and MERS-CoV [2].

In-duct ultraviolet germicidal irradiation (UVGI) systems treat moving air streams in HVAC systems to inactivate airborne microorganisms. UVGI system performance and costs to implement and operate the system depend greatly on the output of the UV lamps and the exposure time, which are affected by the temperature and the velocity of the air passing through the UVGI device [9]. The UV lights cannot be installed inside the fan-coils, without other measures that increase their effectiveness, due to the short exposure of the air during the crossing.

UV irradiation is also used with $\mathrm{TiO}_{2}$ particles and causes photocatalytic antimicrobial reactions. $\mathrm{TiO}_{2}$ photocatalysis induced by $\mathrm{UV}$ irradiation generates strong hydroxyl radicals $(\cdot \mathrm{OH})$, superoxide ions $\left(\mathrm{O}_{2}{ }^{-}\right)$, and hydrogen peroxide $\left(\mathrm{H}_{2} \mathrm{O}_{2}\right)$, which are capable of 
inactivating waterborne and airborne microorganisms. It was reported that the penetration efficiency of Legionella pneumophila through photocatalytic filters with UV irradiation was more than four times smaller than that through normal filters [8]. In a test, three $18 \mathrm{~W}$ fluorescent visible white-light lamps with a $\mathrm{TiO}_{2}$ coating of $5.9 \mathrm{~g}$ could reduce $9-84 \%$ of culturable bacteria bioaerosols and $3-74 \%$ of culturable fungal bioaerosols within 30-480 min of irradiation. The effectiveness of photocatalysis depends greatly on the concentration of oxidant radicals, which is a function of the reaction range of UV irradiation, the amount of $\mathrm{TiO}_{2}$ particles, and the operating time.

\subsection{Tests conducted on fan-coils}

Aermec has been using photocatalytic filters for several years, installed inside its fancoils, as shown in figure 7. The use of UV light allows both the titanium plate and the air to be irradiated directly. In 2016, tests were conducted with the chemistry department and the pharmacy school of the University of Camerino, which demonstrated an almost total cancellation of the bacterium staphylococcus epidermidis after only 90 minutes, a reduction of $80 \%$ of VOCs in 4 hours, a similar abatement for formaldehyde and benzene.
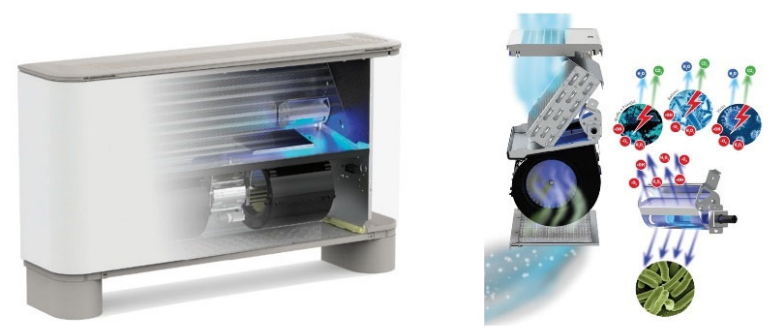

Fig. 7 - Position of photocatalytic filters inside the fan-coil

Following the Sars-COV-2 pandemic, the need was felt to test the effectiveness of the system also on the virus that triggered the pandemic. The studies were conducted with the Department of Microbiology of the University of Padua. The tests were conducted on viruses positioned on the surfaces, both for the difficulty of carrying out tests directly on the aerosol, and to demonstrate that the effectiveness of the system is not only in cleaning the air, but also in disinfecting the entire room surfaces included, which is impossible for all other filters. The test was conducted with an air temperature in the chamber of $20^{\circ} \mathrm{C}$.

The SARS-COV-2 virus is much more resistant on surfaces than in the air. (Magurano and others [10] have shown that, for air temperatures below $28^{\circ} \mathrm{C}$, viral loads remain constant for at least 8 hours on many surfaces, to decrease slightly after 12 hours, reduce up to $10 \%$ after 24 hours and cancel completely after more than 48 hours.

The tests conducted confirmed this trend (figure 8, left graph). The effect of the photocatalytic filter installed in the fan-coil follows this trend, increasing it beyond the eighth hour. The graph on the right shows the trend of the filter efficiency as a function of both time and fan speed. For the minimum speed, only the measurement after 12 hours was carried out. The efficiency is constant in the first 8 hours and then increases to the twelfth, when the virus begins to reduce naturally.

Considering the excellent results obtained on the viral loads present on the surfaces, a test campaign is being organized directly on the aerosol, much more complex to conduct. 

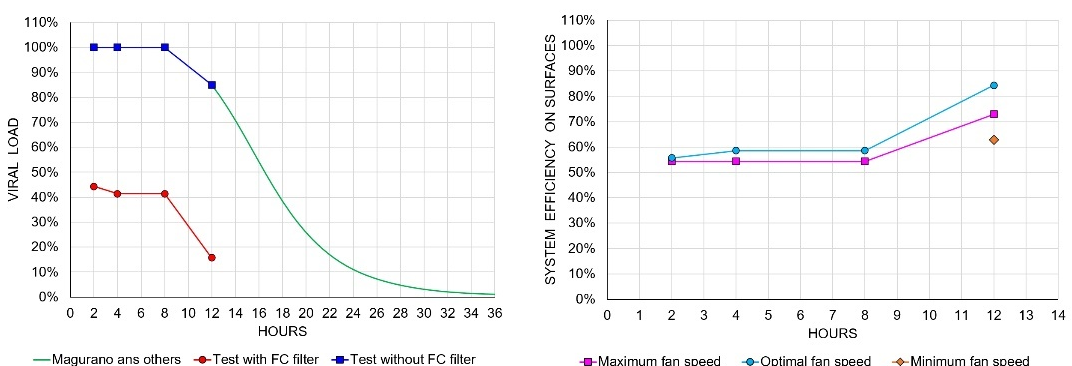

Fig. 8 - Results of tests conducted at the Department of Microbiology of the University of Padua

The efficiency of photocatalytic filters is influenced by the fan speed. The maximum value occurs at an optimal speed, intermediate between the maximum and the minimum. This is easily explained: at the minimum speed there is a lower transport of the particles released from the system, capable of inhibiting the virus, while at maximum speed their concentration is reduced. Therefore, the optimal speed is the one able to guarantee the maximum concentration of the particles released by the photocatalytic system. However, mechanical and electrostatic filters also have a similar behaviour.

Effects on aerosol are believed to be better for at least three reasons:

- the life of the virus in the aerosol is significantly lower (Van Doremalen and others, 2020). The tests on the surfaces have shown an increase in efficiency when the virus begins to decay naturally: it is presumed that this also happens for the viral loads contained in the aerosol and that, therefore, a high efficiency is reached extremely quickly;

- $\quad$ the air is however irradiated by the UV light inside the fan-coil: even if its power is not high, there is still an reduction effect on the virus life;

- the action of the photocatalytic filter in the air is greater than that on the surfaces, because there is a greater mixing between the viral loads to be eliminated and hydroxyl radicals, superoxide ions and hydrogen peroxide released into the air.

\section{Conclusion}

In the case of airborne diseases, the recirculation of the air within a single room, or between several different rooms, can be an excellent tool to always reduce the maximum individual risk of infection, because it spreads the virus in a greater volume, reducing the maximum concentration, and also the collective risk of infection, with an appropriate filtration.

In total air systems it is absolutely not necessary to use absolute filters, even if the AHUs are able to house them: satisfactory results are achieved already with a filtration efficiency between $75 \%$ and $85 \%$ on the particles that make up the aerosol.

In the case of primary air systems, the most promising filtration system is the photocatalytic one which allows not only filtration, but also cleaning the air because it kills viruses, germs and bacteria very quickly and with high efficiency. 


\section{References}

1. Ai Z.T., Melikov A.K. Airborne spread of expiratory droplet nuclei between the occupants of indoor environments: A review. Indoor Air 28, 500-524. (2018)

2. Bedell K, Buchaklian AH, Perlman S. Efficacy of an automated multiple emitter wholeroom ultraviolet-C disinfection system against coronaviruses MHV and MERS-CoV. Infect Control Hosp Epidemiol 37: 598 -599 (2016)

3. Buonanno G., Morawska L. Stabile, L.. Quantitative assessment of the risk of airborne transmission of SARS-CoV-2 infection: Prospective and retrospective applications. Environmental International, 45, art. n. 106112 (2020)

4. Buonanno G., d'Ambrosio F.R., Strada M., Vio M.: La climatizzazione dell'aria: riflessioni suggerite dalla Covid 19, Editoriale Delfino (2021)

5. Dietz L., Horve P., Coil D., Fretz M., Eisen J, Van Den Wymelenberga K: 2019 Novel Coronavirus (COVID-19) Pandemic: Built Environment Considerations To Reduce Transmission, American society for microbiology, Volume 5 (2020)

6. Gammaitoni L., Nucci M.C. Using a mathematical model to evaluate the efficacy of TB control measures. Emerging Infectious Diseases, 335-342 (1997)

7. Knibbs L.D., Morawska L., Bell S.C., Grzybowsky P. Room ventilation and the risk of airborne innfection transmission in health care settings within a large teaching hospital. American Journal of Infection Control, 39(10), 866-872 (2011)

8. Lee B. U.: Life Comes from the Air: A Short Review on Bioaerosol Control, Aerosol and Air Quality Research, 11: 921-927 (2011)

9. Lee B. Bahnfleth W.P.: Effects of installation location on performance and economics of in-duct ultraviolet germicidal irradiation systems for air disinfection, Building and environment 67, 193-201 (2013)

10. Magurano F., Baggieri M., Marchi A., Rezza G., Nicoletti L. : SARS-CoV-2 infection: the environmental endurance of the virus can be influenced by the increase of temperature, Clinical Microbiology and Infection 27, 289.e5 - 289.e7 (2021)

11. Riley EC, Murphy G, Riley RL. Airborne spread of measles in a suburban elementary school. Am J Epidemiol; 107:421-32 (1978)

12. Schuit M, Gardner S, Wood S, Bower K, Williams G, Freeburger D, Dabisch P. The influence of simulated sunlight on the inactivation of influenza virus in aerosols. J Infect Dis 221:372-378 (2020)

13. van Doremalen N., Bushmaker T. and oth... Aerosol and Surface Stability of SARSCoV-2 as Compared with SARS-CoV-1. N Engl J Med. (2021). 